it was hence found to decrease. From the obtained dependence on $d$, the $\lambda_{\mathrm{S}}$, at $2.4 \mathrm{eV}$ above the highest occupied molecular orbital level of the OSEC was determined to be $\sim 13 \mathrm{~nm}$ in $\mathrm{CuPc}$ at room temperature.

The works of Drew et al. ${ }^{9}$ and Cinchetti et al. ${ }^{10}$ both attest that SP carriers are indeed injected in FM-OSEC junctions, thus validating previous experiments performed on organic spin valves using $\operatorname{MR}\left(B_{0}\right)$ dependence ${ }^{6}$. It should be said, however, that the two techniques described are rather complicated, require sophisticated analysis to obtain meaningful results and, most importantly, are not 'tabletop' type techniques. To obtain a technique comparable in accessibility to those used for inorganic materials ${ }^{11,12}$ - based, for example, on the detection of circularly polarized electroluminescence - it is essential to find an OSEC material with a sufficiently strong spin-orbit interaction that allows implementation of such circular polarization spectroscopic techniques. At present, however, this would mean that such an organic material, if found, would have a limited $\lambda_{\mathrm{s}}$ owing to the unavoidable enhanced spin-orbit coupling. A solution would be to find a material that also has high carrier mobility, which by compensating the spin relaxation due to the spin-orbit coupling would yield $\lambda_{\mathrm{S}}$ on the order of tens of nanometres at room temperature.

A final point that should be mentioned is that the two works raise important questions about the temperature dependence of $\lambda_{\mathrm{s}}$ in OSEC. Namely, what is the origin of the spin-flip processes that were found? And what is the temperature dependence of these processes? These questions are crucial, and may determine the ability to use OSEC spintronics devices at room temperature ${ }^{5,14}$.
Z. Valy Vardeny is in the Department of

Physics, University of Utah, Salt Lake City,

Utah 84112, USA.

e-mail:val@physics.utah.edu

References

1. Wolf, S. A. et al. Science 294, 1488-1495 (2001)

2. Zutic, I., Fabian, J. \& Das Sarma, S. Rev. Mod. Phys. 76, 323-410 (2004)

3. Dediu, V. et al. Solid State Commun. 122, 181-184 (2002)

4. Pramanik, S. et al. Nature Nanotech. 2, 216-219 (2007).

5. Sanvito, S. Nature Mater. 6, 803-804 (2007).

6. Xiong, Z. H., Wu, D., Vardeny, Z. V. \& Shi, J. Nature 427, 821-824 (2004).

Xu, W. et al. Appl. Phys. Lett. 90, 072506 (2007)

8. Jiang, J. S., Pearson, J. E. \& Bader, S. D. Phys. Rev. B 77, 035303 (2008)

9. Drew, A. J. et al. Nature Mater. 8, 109-114 (2009).

10. Cinchetti, M. et al. Nature Mater. 8, 115-119 (2009).

11. Kikkawa, J. M. \& Awschalom D. D. Nature 397, 139-141 (1999).

12. Crooker, S. A. et al. Science 309, 2191-2196 (2005).

13. Bakule, P. \& Morenzoni, E. Contemp. Phys. 45, 203-225 (2004).

14. Yunus, M., Ruden, P. P. \& Smith D. L. Appl. Phys. Lett. 93, $123312(2008)$

\title{
WHAT'S PHILOSOPHY GOT TO DO WITH IT?
}

"Should engineers think?" That question, at first glance mildly insulting to the profession, crops up early in a recent volume of Interdisciplinary Science Reviews devoted to 'Philosophy and Engineering' (33(3), 2008). But as Natasha McCarthy of the UK's Royal Academy of Engineering points out in her editorial introduction, "The stereotype tells us that engineers are 'doers' and not 'thinkers.'

Leaving aside the flaws in the notion of sharp boundaries between scientists (the 'thinkers') and engineers, the message is clear: engineers have a job to do, and 'philosophy' is rarely seen as relevant to it. But beyond thinking about the technical aspects of the task, should engineers consider matters of cost, safety, risk, environmental, social, ethical and aesthetic impacts? If they don't, should this be seen as a form of engineering failure?

Of course, few engineering jobs don't explicitly embrace some of these concerns. Many materials, for example, are developed specifically to improve safety or to reduce harm to the environment. But even then, the issues are not always straightforward. A biodegradable product can't be assumed to be 'greener' without a life-cycle analysis that might lie beyond the expertise of the product designers. No one foresaw, or could be expected to foresee, or even now agrees on, the social impact of automobiles, computers and mobile phones.

But truly to engage with some of these matters, or even to make seemingly routine assessments of risk and safety, might require that engineers acknowledge a genuinely philosophical dimension of the profession, particularly in the sphere of epistemology, or as McCarthy puts it, what we know and how we know what we know. Partly this is a question of how one obtains reliable information. But there has been increasing recognition that it also bears on what is knowable. Some complex engineered systems show emergent phenomena that can't be predicted, even in principle, from knowledge of the components. Dealing with that challenge is considered here by W. P. S. Dias of the University of Moratuwa in Sri Lanka, and by Darryl Farber and colleagues at Pennsylvania State University. A related difficulty tackled by the latter group is how to meaningfully forecast performance in the face of incomplete knowledge about what the system will encounter.

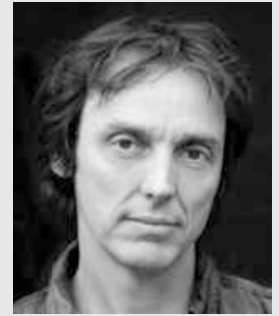

PHILIP BALL

Epistemology aside, much of engineering philosophy might be considered to encompass ethics. Here McCarthy takes a stance: an engineer cannot be expected to reflect on aspects beyond the call of duty, which basically means getting the job done efficiently, effectively and safely. Indeed, it might be dangerous to do so without the necessary expertise. But that, she says, doesn't absolve engineers of moral obligations for they have a duty to ensure that such broader issues have been given due thought by others suitably qualified.

This sound principle might quickly become a minefield in practice. How can engineers assure themselves that this process has been carried out, and done well? How is the obligation enforced? (Clearly, it isn't.) In this and other ways, this volume is just the start of the discussion. 